\title{
Discrimination Between B-Processes is Impossible
}

\author{
Daniil Ryabko
}

Received: 29 August 2008 / Revised: 3 November 2009 / Published online: 2 December 2009

(C) Springer Science+Business Media, LLC 2009

\begin{abstract}
Two series of binary observations $x_{1}, x_{1}, \ldots$ and $y_{1}, y_{2}, \ldots$ are presented: $x_{n}$ and $y_{n}$ are given at each time $n \in \mathbb{N}$. It is assumed that the sequences are generated independently of each other by two B-processes. The question of interest is whether the sequences represent a typical realization of two different processes or of the same one. It is demonstrated that this is impossible to decide, in the sense that every discrimination procedure is bound to err with non-negligible frequency when presented with sequences from some $B$-processes. This contrasts with earlier positive results on $B$-processes, in particular, those showing that there are consistent $\bar{d}$-distance estimates for this class of processes, and on ergodic processes, in particular, those establishing consistent change point estimates.
\end{abstract}

Keywords Process discrimination $\cdot B$-processes $\cdot$ Stationary ergodic processes · Time series $\cdot$ Homogeneity testing

Mathematics Subject Classification (2000) Primary 62G10 • 60G10 · Secondary $62 \mathrm{M} 07 \cdot 37 \mathrm{~A} 99 \cdot 60 \mathrm{~J} 10$

\section{Introduction}

Two series of binary observations $x_{1}, x_{1}, \ldots$ and $y_{1}, y_{2}, \ldots$ are presented sequentially. A discrimination procedure $D$ is a family of mappings $D_{n}: X^{n} \times X^{n} \rightarrow\{0,1\}$, $n \in \mathbb{N}, X=\{0,1\}$, that maps a pair of samples $\left(x_{1}, \ldots, x_{n}\right),\left(y_{1}, \ldots, y_{n}\right)$ into a binary ("yes" or "no") answer: the samples are generated by different distributions, or they are generated by the same distribution.

A discrimination procedure $D$ is asymptotically correct for a set $\mathcal{C}$ of process distributions if, for any two distributions $\rho_{x}, \rho_{y} \in \mathcal{C}$ independently generating the

D. Ryabko (西)

INRIA Lille, 40 avenue Halley, 59650 Villeneuve d'Ascq, France

e-mail: daniil@ryabko.net 
sequences $x_{1}, x_{2}, \ldots$ and $y_{1}, y_{2}, \ldots$, respectively, the expected output converges to the correct answer: the following limit exists, and the equality holds:

$$
\lim _{n \rightarrow \infty} \mathbf{E} D_{n}\left(\left(x_{1}, \ldots, x_{n}\right),\left(y_{1}, \ldots, y_{n}\right)\right)= \begin{cases}0 & \text { if } \rho_{x}=\rho_{y}, \\ 1 & \text { otherwise }\end{cases}
$$

This is perhaps the weakest notion of correctness one can consider. Clearly, asymptotically correct discriminating procedures exist for many classes of processes, for example, for the class of all i.i.d. processes (e.g., [4]) and various parametric families.

We show that there is no asymptotically correct discrimination procedure for the class of all $B$-processes (see the definition below), meaning that for any discrimination, the expected answer does not converge to the correct one for some processes. The class of $B$-processes is sufficiently wide to include, for example, $k$-order Markov processes and functions of them, but, on the other hand, it is a strict subset of the set of stationary ergodic processes. $B$-processes play an important role in such fields as information theory and ergodic theory $[7,15,16]$.

Previously, Ornstein and Weiss [9] and Ornstein and Shields [8] showed that consistent estimates of $\bar{d}$-distance (defined below) for $B$-processes exist, while it is impossible to estimate this distance outside this class. The latter result, as well as the result of the present work, contrasts with the positive results of $[6,11-13]$, which show, in particular, that asymptotically consistent change-point estimation is possible for stationary ergodic real-valued processes. Thus, we can say that discrimination is harder than distance estimation and change-point estimation. The result of this work also complements earlier negative results on $B$-processes and stationary ergodic processes, such as $[1,2,5,10,14]$, that establish negative results concerning prediction, density estimation, testing membership to certain families of processes, and others. The construction used in the proof of the result of this work is somewhat similar to that of [1] used to show that consistent density estimation is impossible for stationary ergodic processes (although the latter uses the method of cutting and stacking rather than Markov chains employed here).

Next, we define the $\bar{d}$ distance and $B$-processes (mainly following [9] in our formulations) and give more precise formulations of some of the existing results mentioned above. The main result of this work is formulated and proven in the next section.

For two finite-valued stationary processes $\rho_{x}$ and $\rho_{y}$, the $\bar{d}$-distance $\bar{d}\left(\rho_{x}, \rho_{y}\right)$ is said to be less than $\varepsilon$ if there exists a single stationary process $v_{x y}$ on pairs $\left(x_{n}, y_{n}\right)$, $n \in \mathbb{N}$, such that $x_{n}, n \in \mathbb{N}$, are distributed according to $\rho_{x}$, and $y_{n}$ are distributed according to $\rho_{y}$, while

$$
v_{x y}\left(x_{1} \neq y_{1}\right) \leq \varepsilon .
$$

The infimum of the $\varepsilon$ 's for which a coupling can be found such that (1) is satisfied is taken to be the $\bar{d}$-distance between $\rho_{x}$ and $\rho_{y}$.

A process is called a $B$-process (or a Bernoulli process) if it is in the $\bar{d}$-closure of the set of all aperiodic stationary ergodic $k$-step Markov processes, where $k \in \mathbb{N}$. For more information on $\bar{d}$-distance and $B$-processes, the reader is referred to [7]. 
As it was mentioned, [9] constructs an estimator $\bar{s}_{n}$ such that

$$
\lim _{n \rightarrow \infty} \bar{s}_{n}\left(\left(x_{1}, \ldots, x_{n}\right),\left(y_{1}, \ldots, y_{n}\right)\right)=\bar{d}\left(\rho_{1}, \rho_{2}\right) \quad \rho_{1} \times \rho_{2} \text {-a.s. }
$$

if both processes $\rho_{1}$ and $\rho_{2}$ generating the samples $x_{i}$ and $y_{i}$, respectively, are $B$-processes. In the same work it is shown that there is no estimator $\bar{s}_{n}$ for which (2) holds for every pair $\rho_{1}, \rho_{2}$ of stationary ergodic processes. Some extensions of these results are given in [8].

It is interesting to compare these results to those that are obtained for a weaker process distance, the distributional distance. (As far as the results of the present work are concerned, this distance is only used in the proof.) It is defined as follows. Denote by $X^{*}$ the set of all finite tuples $X^{*}:=\bigcup_{k \in \mathbb{N}} X^{k}$. Assuming length-lexicographical order on $X^{*}$, introduce the notation $X^{*}=\left\{B_{1}, B_{2}, \ldots\right\}$ for the elements of this set, and let $\left|B_{i}\right|$ denote the length of $B_{i}$ (that is, $\left|B_{i}\right|=k$ if $B_{i} \in X^{k}$ ). Furthermore, define the weights $w_{k}:=2^{-k}$. For (arbitrary) process distributions $\rho_{1}, \rho_{2}$, the distributional distance $d\left(\rho_{1}, \rho_{2}\right)$ between them is defined as

$$
d\left(\rho_{1}, \rho_{2}\right)=\sum_{i=1}^{\infty} w_{i}\left|\rho_{1}\left(\left(x_{1}, \ldots, x_{\left|B_{i}\right|}\right)=B_{i}\right)-\rho_{2}\left(\left(x_{1}, \ldots, x_{\left|B_{i}\right|}\right)=B_{i}\right)\right| .
$$

We refer to [3] for more information on distributional distance and its properties. Notably, this distance is weaker than the $\bar{d}$ distance. In [12, 13] an estimator $\bar{s}_{n}\left(\left(x_{1}, \ldots, x_{n}\right),\left(y_{1}, \ldots, y_{n}\right)\right)$ is constructed such that

$$
\lim _{n \rightarrow \infty} s_{n}\left(\left(x_{1}, \ldots, x_{n}\right),\left(y_{1}, \ldots, y_{n}\right)\right)=d\left(\rho_{1}, \rho_{2}\right) \quad \rho_{1} \times \rho_{2} \text {-a.s. }
$$

if both processes $\rho_{1}$ and $\rho_{2}$ generating the samples $x_{i}$ and $y_{i}$, respectively, are stationary ergodic. This estimator is also used to construct a consistent change-point estimate. That is, given a sample

$$
\left(z_{1}, \ldots, z_{n}\right)=\left(x_{1}, \ldots, x_{\theta n}, y_{\theta n+1}, \ldots, y_{n}\right)
$$

which is a concatenation of two samples generated by two different stationary ergodic processes, with the point of change (concatenation) $\theta \in(0,1)$ being the unknown parameter to estimate, there is an estimator $\hat{\theta}_{n}$ such that $\left|\hat{\theta}_{n}-\theta\right|=o(1)$ almost surely as the size $n$ of the sample goes to infinity. This holds even for real-valued processes [12]. On the other hand, the results of the present work imply that one cannot consistently tell whether there is a change in the sample or not.

Summarizing, we can say that the stronger the distance, the harder it is to estimate: the distributional distance can be consistently estimated for stationary ergodic processes, the $\bar{d}$ distance can be consistently estimated for $B$-processes but not for stationary ergodic processes, while, as is shown in this work, the strongest possible distance - the one that gives discrete topology — cannot be consistently estimated for $B$-processes. 


\section{The Main Result}

The main result of this work is the theorem below. The construction on which the proof is based uses the ideas of the construction of Ryabko [10] to demonstrate that consistent prediction for stationary ergodic processes is impossible (see also the modification of this construction in [2]).

Theorem 1 There is no asymptotically correct discrimination procedure for the set of all B-processes.

Proof We will assume that asymptotically correct discrimination procedure $D$ for the class of all $B$-processes exists and will construct a $B$-process $\rho$ such that if both sequences $x_{i}$ and $y_{i}, i \in \mathbb{N}$, are generated by $\rho$, then $\mathbf{E} D_{n}$ diverges; this contradiction will prove the theorem.

The scheme of the proof is as follows. In Step 1 we construct a sequence of processes $\rho_{2 k}, \rho_{d 2 k+1}$, and $\rho_{u 2 k+1}$, where $k=0,1, \ldots$ In Step 2 we construct a process $\rho$, which is shown to be the limit of the sequence $\rho_{2 k}, k \in \mathbb{N}$, in $\bar{d}$-distance. In Step 3 we show that two independent runs of the process $\rho$ have the property that (with high probability) they first behave like two runs of a single process $\rho_{0}$, then like two runs of two different processes $\rho_{u 1}$ and $\rho_{d 1}$, then like two runs of a single process $\rho_{2}$, and so on, thereby showing that the test $D$ diverges and obtaining the desired contradiction.

Assume that there exists an asymptotically correct discriminating procedure $D$. Fix some $\varepsilon \in(0,1 / 2)$ and $\delta \in[1 / 2,1)$ to be defined in Step 3 .

Step 1 . We will construct the sequence of process $\rho_{2 k}, \rho_{u 2 k+1}$, and $\rho_{d 2 k+1}$, where $k=0,1, \ldots$.

Step 1.0. Construct the process $\rho_{0}$ as follows. A Markov chain $m_{0}$ is defined on the set $\mathbb{N}$ of states. From each state $i \in \mathbb{N}$ the chain passes to the state 0 with probability $\delta$ and to the state $i+1$ with probability $1-\delta$. With transition probabilities so defined, the chain possesses a unique stationary distribution $M_{0}$ on the set $\mathbb{N}$, which can be calculated explicitly by using, e.g., [17, Theorem VIII.4.1] and is as follows: $M_{0}(0)=\delta$ and $M_{0}(k)=\delta(1-\delta)^{k}$ for all $k \in \mathbb{N}$. Take this distribution as the initial distribution over the states.

The function $f_{0}$ maps the states to the output alphabet $\{0,1\}$ as follows: $f_{0}(i)=1$ for every $i \in \mathbb{N}$. Let $s_{t}$ be the state of the chain at time $t$. The process $\rho_{0}$ is defined as $\rho_{0}=f_{0}\left(s_{t}\right)$ for $t \in \mathbb{N}$. As a result of this definition, the process $\rho_{0}$ simply outputs 1 with probability 1 on every time step (however, by using different functions $f$ we will have less trivial processes in the sequel). Clearly, the constructed process is stationary ergodic and a $B$-process. So, we have defined the chain $m_{0}$ (and the process $\rho_{0}$ ) up to a parameter $\delta$.

Step 1.1. We begin with the process $\rho_{0}$ and the chain $m_{0}$ of the previous step. Since the test $\mathrm{D}$ is asymptotically correct, we have

$$
\mathbf{E}_{\rho_{0} \times \rho_{0}} D_{t_{0}}\left(\left(x_{1}, \ldots, x_{t_{0}}\right),\left(y_{1}, \ldots, y_{t_{0}}\right)\right)<\varepsilon
$$

from some $t_{0}$ on, where both samples $x_{i}$ and $y_{i}$ are generated by $\rho_{0}$ (that is, both samples consist of $1 \mathrm{~s}$ only). Let $k_{0}$ be such an index that the chain $m_{0}$ starting from 
the state 0 with probability 1 does not reach the state $k_{0}-1$ by time $t_{0}$ (we can take $\left.k_{0}=t_{0}+2\right)$.

Construct two processes $\rho_{u 1}$ and $\rho_{d 1}$ as follows. They are also based on the Markov chain $m_{0}$, but the functions $f$ are different. The function $f_{u 1}: \mathbb{N} \rightarrow\{0,1\}$ is defined as follows: $f_{u 1}(i)=f_{0}(i)=1$ for $i \leq k_{0}$ and $f_{u 1}(i)=0$ for $i>k_{0}$. The function $f_{d 1}$ is identically $1\left(f_{d 1}(i)=1, i \in \mathbb{N}\right)$. The processes $\rho_{u 1}$ and $\rho_{d 1}$ are defined as $\rho_{u 1}=f_{u 1}\left(s_{t}\right)$ and $\rho_{d 1}=f_{d 1}\left(s_{t}\right)$ for $t \in \mathbb{N}$. Thus the process $\rho_{d 1}$ will again produce only $1 \mathrm{~s}$, but the process $\rho_{u 1}$ will occasionally produce $0 \mathrm{~s}$.

Step 1.2. Being run on two samples generated by the processes $\rho_{u 1}$ and $\rho_{d 1}$ which both start from the state 0 , the test $D_{n}$ in the first $t_{0}$ steps produces many $0 \mathrm{~s}$, since on these first $k_{0}$ states all the functions $f, f_{u 1}$, and $f_{d 1}$ coincide. However, since the processes are different and the test is asymptotically correct (by assumption), the test starts producing $1 \mathrm{~s}$, until by a certain time step $t_{1}$ almost all answers are $1 \mathrm{~s}$. Next we will construct the process $\rho_{2}$ by "gluing" together $\rho_{u 1}$ and $\rho_{d 1}$ and continuing them in such a way that, being run on two samples produced by $\rho_{2}$, the test first produces 0 s (as if the samples were drawn from $\rho_{0}$ ), and then, with probability close to $1 / 2$, it produces many 1s (as if the samples were from $\rho_{u 1}$ and $\rho_{d 1}$ ) and then again 0s.

The process $\rho_{2}$ is the pivotal point of the construction, so we give it in some detail. In step $1.2 \mathrm{a}$ we present the construction of the process, and in step $1.2 \mathrm{~b}$ we show that this process is a $B$-process by demonstrating that it is equivalent to a (deterministic) function of a Markov chain.

Step 1.2a. Let $t_{1}>t_{0}$ be a time index such that

$$
\mathbf{E}_{\rho_{u 1} \times \rho_{d 1}} D_{k}\left(\left(x_{1}, \ldots, x_{t_{1}}\right),\left(y_{1}, \ldots, y_{t_{1}}\right)\right)>1-\varepsilon,
$$

where the samples $x_{i}$ and $y_{i}$ are generated by $\rho_{u 1}$ and $\rho_{d 1}$, respectively, (the samples are generated independently; that is, the processes are based on two independent copies of the Markov chain $m_{0}$ ). Let $k_{1}>k_{0}$ be an index such that the chain $m$ starting from the state 0 with probability 1 does not reach the state $k_{1}-1$ by time $t_{1}$.

Construct the process $\rho_{2}$ as follows (see Fig. 1). It is based on a chain $m_{2}$ on which Markov assumption is violated. The transition probabilities on states $0, \ldots, k_{0}$ are the same as for the Markov chain $m$ (from each state return to 0 with probability $\delta$ or go to the next state with probability $1-\delta$ ).

There are two "special" states, the "switch" $S_{2}$ and the "reset" $R_{2}$. From the state $k_{0}$ the chain passes with probability $1-\delta$ to the "switch" state $S_{2}$. The switch $S_{2}$ can itself have two values, up and down. If $S_{2}$ has the value up, then from $S_{2}$ the chain passes to the state $u_{k_{0}+1}$ with probability 1 , while if $S_{2}=d o w n$, the chain goes to $d_{k_{0}+1}$, with probability 1 . If the chain reaches the state $R_{2}$, then the value of $S_{2}$ is set to $u p$ with probability $1 / 2$, and with probability $1 / 2$ it is set to down. In other words, the first transition from $S_{2}$ is random (either to $u_{k_{0}+1}$ or to $d_{k_{0}+1}$ with equal probabilities), and then this decision is remembered until the "reset" state $R_{2}$ is visited, whereupon the switch again assumes the values up and down with equal probabilities.

The rest of the transitions are as follows. From each state $u_{i}, k_{0} \leq i \leq k_{1}$ the chain passes to the state 0 with probability $\delta$ and to the next state $u_{i+1}$ with probability $1-\delta$. From the state $u_{k_{1}}$ the process goes with probability $\delta$ to 0 and with probability $1-\delta$ to the "reset" state $R_{2}$. The same with states $d_{i}$ : for $k_{0}<i \leq k_{1}$, the process 


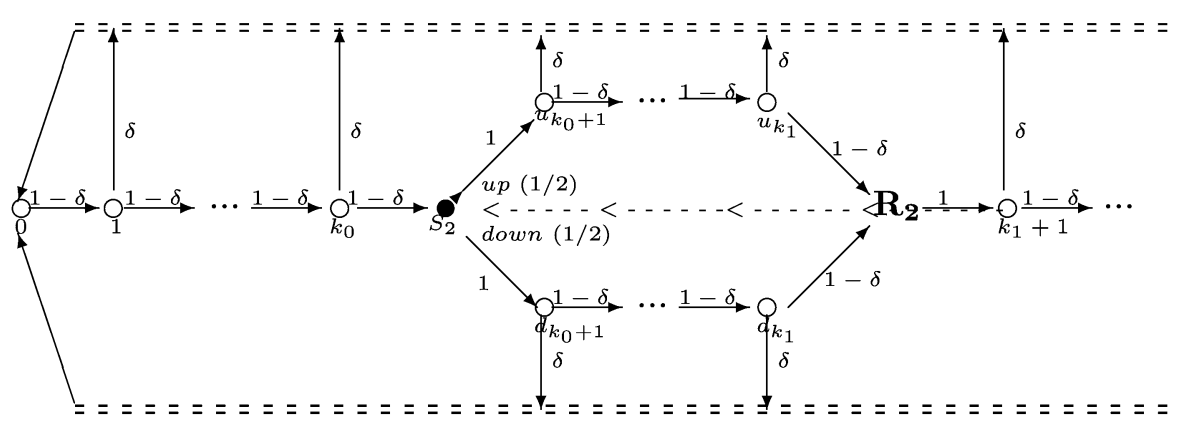

Fig. 1 The processes $m_{2}$ and $\rho_{2}$. The states are depicted as circles, the arrows symbolize transition probabilities: from every state the process returns to 0 with probability $\delta$ or goes to the next state with probability $1-\delta$. From the switch $S_{2}$ the process passes to the state indicated by the switch (with probability 1 ); here it is the state $u_{k_{0}+1}$. When the process passes through the reset $R_{2}$, the switch $S_{2}$ is set to either up or down with equal probabilities. (Here $S_{2}$ is in the position up.) The function $f_{2}$ is 1 on all states except $u_{k 0+1}, \ldots, u_{k 1}$ where it is $0 ; f_{2}$ applied to the states output by $m_{2}$ defines $\rho_{2}$

returns to 0 with probability $\delta$ or goes to the next state $d_{i+1}$ with probability $1-\delta$, where the next state for $d_{k_{1}}$ is the "reset" state $R_{2}$. From $R_{2}$ the process goes with probability 1 to the state $k_{1}+1$ where from the chain continues ad infinitum: to the state 0 with probability $\delta$ or to the next state $k_{1}+2$ with probability $1-\delta$, etc.

The initial distribution on the states is defined as follows. The probabilities of the states $0, \ldots, k_{0}, k_{1}+1, k_{1}+2, \ldots$ are the same as in the Markov chain $m_{0}$, that is, $\delta(1-\delta)^{j}$ for $j=0, \ldots, k_{0}, k_{1}+1, k_{1}+2, \ldots$. For the states $u_{j}$ and $d_{j}, k_{0}<j \leq k_{1}$, define their initial probabilities to be $1 / 2$ of the probability of the corresponding state in the chain $m_{0}$, that is, $m_{2}\left(u_{j}\right)=m_{2}\left(d_{j}\right)=m_{0}(j) / 2=\delta(1-\delta)^{j} / 2$. Furthermore, if the chain starts in a state $u_{j}, k_{0}<j \leq k_{1}$, then the value of the switch $S_{2}$ is $u p$, and if it starts in the state $d_{j}$, then the value of the switch $S_{2}$ is down, whereas if the chain starts in any other state, then the probability distribution on the values of the switch $S_{2}$ is $1 / 2$ for either up or down.

The function $f_{2}$ is defined as follows: $f_{2}(i)=1$ for $0 \leq i \leq k_{0}$ and $i>k_{1}$ (before the switch and after the reset); $f_{2}\left(u_{i}\right)=0$ for all $i, k_{0}<i \leq k_{1}$, and $f_{2}\left(d_{i}\right)=1$ for all $i, k_{0}<i \leq k_{1}$. The function $f_{2}$ is undefined on $S_{2}$ and $R_{2}$, and therefore there is no output on these states (we also assume that passing through $S_{2}$ and $R_{2}$ does not increment time). As before, the process $\rho_{2}$ is defined as $\rho_{2}=f_{2}\left(s_{t}\right)$, where $s_{t}$ is the state of $m_{2}$ at time $t$, omitting the states $S_{2}$ and $R_{2}$. The resulting process is illustrated in Fig. 1.

Step 1.2b. To show that the process $\rho_{2}$ is stationary ergodic and a $B$-process, we will show that it is equivalent to a function of a stationary ergodic Markov chain, whereas all such process are known to be $B$ (e.g., [16]). The construction is as follows (see Fig. 2). This chain has states $k_{1}+1, \ldots$ and also $u_{0}, \ldots, u_{k_{0}}, u_{k_{0}+1}, \ldots, u_{k_{1}}$ and $d_{0}, \ldots, d_{k_{0}}, d_{k_{0}+1}, \ldots, d_{k_{1}}$. From the states $u_{i}, i=0, \ldots, k_{1}$, the chain passes with probability $1-\delta$ to the next state $u_{i+1}$, where the next state for $u_{k_{1}}$ is $k+1$ and with probability $\delta$ returns to the state $u_{0}$ (and not to the state 0 ). Transitions for the state $d_{0}, \ldots, d_{k_{1}-1}$ are defined analogously. Thus the states $u_{k_{i}}$ correspond to the state $u p$ of the switch $S_{2}$ and the states $d_{k_{i}}$ to the state down of the switch. Transitions for the states $k+1, k+2, \ldots$ are defined as follows: with probability $\delta / 2$ to the state $u_{0}$, 


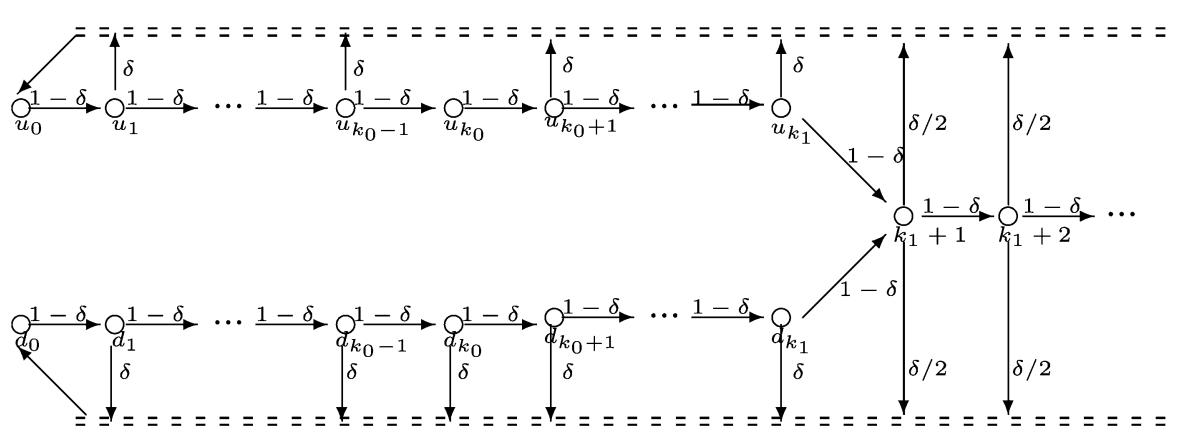

Fig. 2 The process $m_{2}^{\prime}$. The function $f_{2}$ is 1 everywhere except the states $u_{k_{0}+1}, \ldots, u_{k_{1}}$, where it is 0

with probability $\delta / 2$ to the state $d_{0}$, and with probability $1-\delta$ to the next state. Thus, transitions to 0 from the states with indices greater than $k_{1}$ correspond to the reset $R_{2}$. Clearly, the chain $m_{2}^{\prime}$ as defined possesses a unique stationary distribution $M_{2}$ over the set of states and $M_{2}(i)>0$ for every state $i$. Moreover, this distribution is the same as the initial distribution on the states of the chain $m_{0}$, except for the states $u_{i}$ and $d_{i}$, for which we have $m_{2}^{\prime}\left(u_{i}\right)=m_{2}^{\prime}\left(d_{i}\right)=m_{0}(i) / 2=\delta(1-\delta)^{i} / 2$ for $0 \leq i \leq k_{0}$. We take this distribution as its initial distribution on the states of $m_{2}^{\prime}$. The resulting process $m_{2}^{\prime}$ is stationary ergodic and a $B$-process, since it is a function of a Markov chain [16]. It is easy to see that if we define the function $f_{2}$ on the states of $m_{2}^{\prime}$ as 1 on all states except $u_{k_{0}+1}, \ldots, u_{k_{1}}$, then the resulting process is exactly the process $\rho_{2}$. Therefore, $\rho_{2}$ is stationary ergodic and a $B$-process.

Step 1.k. As before, we can continue the construction of the processes $\rho_{u 3}$ and $\rho_{d 3}$ that start with a segment of $\rho_{2}$. Let $t_{2}>t_{1}$ be a time index such that

$$
\mathbf{E}_{\rho_{2} \times \rho_{2}} D_{t_{2}}<\varepsilon
$$

where both samples are generated by $\rho_{2}$. Let $k_{2}>k_{1}$ be an index such that, when starting from the state 0 , the process $m_{2}$ with probability 1 does not reach $k_{2}-1$ by time $t_{2}$ (equivalently: the process $m_{2}^{\prime}$ does not reach $k_{2}-1$ when starting from either $u_{0}$ or $d_{0}$ ). The processes $\rho_{u 3}$ and $\rho_{d 3}$ are based on the same process $m_{2}$ as $\rho_{2}$. The functions $f_{u 3}$ and $f_{d 3}$ coincide with $f_{2}$ on all states up to the state $k_{2}$ (including the states $u_{i}$ and $\left.d_{i}, k_{0}<i \leq k_{1}\right)$. After $k_{2}$ the function $f_{u 3}$ outputs 0 s, while $f_{d 3}$ outputs 1s: $f_{u 3}(i)=0$ and $f_{d 3}(i)=1$ for $i>k_{2}$.

Furthermore, we find a time $t_{3}>t_{2}$ by which we have $\mathbf{E}_{\rho_{u 3} \times \rho_{d 3}} D_{t_{3}}>1-\varepsilon$, where the samples are generated by $\rho_{u 3}$ and $\rho_{d 3}$, which is possible since $D$ is consistent. Next, find an index $k_{3}>k_{2}$ such that the process $m_{2}$ does not reach $k_{3}-1$ with probability 1 if the processes $\rho_{u 3}$ and $\rho_{d 3}$ are used to produce two independent sequences and both start from the state 0 . We then construct the process $\rho_{4}$ based on a (non-Markovian) process $m_{4}$ by "gluing" together $\rho_{u 3}$ and $\rho_{d 3}$ after the step $k_{3}$ with a switch $S_{4}$ and a reset $R_{4}$ exactly as was done when constructing the process $\rho_{2}$. The process $m_{4}$ is illustrated in Fig. 3a). The process $m_{4}$ can be shown to be equivalent to a Markov chain $m_{4}^{\prime}$, which is constructed analogously to the chain $m_{2}^{\prime}$ (see Fig. 3b). Thus, the process $\rho_{4}$ can be shown to be a $B$-process. 
a)

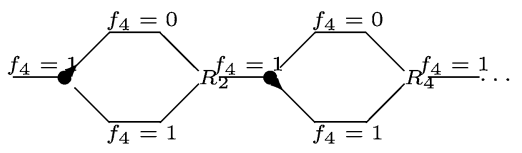

b)

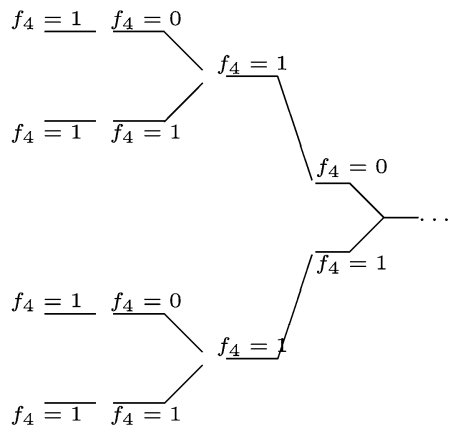

Fig. 3 (a) The processes $m_{4}$. (b) The Markov chain $m_{4}^{\prime}$

Proceeding this way, we can construct the processes $\rho_{2 j}, \rho_{u 2 j+1}$, and $\rho_{d 2 j+1}$, $j \in \mathbb{N}$, choosing the time steps $t_{j}>t_{j-1}$ so that the expected output of the test approaches 0 by the time $t_{j}$ being run on two samples produced by $\rho_{j}$ for even $j$, and approaches 1 by the time $t_{j}$ being run on samples produced by $\rho_{u j}$ and $\rho_{d j}$ for odd $j$ :

$$
\mathbf{E}_{\rho_{2 j} \times \rho_{2 j}} D_{t_{2 j}}<\varepsilon
$$

and

$$
\mathbf{E}_{\rho_{u 2 j+1} \times \rho_{d 2 j+1}} D_{t_{2 j+1}}>(1-\varepsilon) .
$$

For each $j$, the number $k_{j}>k_{j-1}$ is selected in a such a way that the state $k_{j}-1$ is not reached (with probability 1) by the time $t_{j}$ when starting from the state 0 . Each of the processes $\rho_{2 j}, \rho_{u 2 j+1}$, and $\rho_{d j 2+1}, j \in \mathbb{N}$, can be shown to be stationary ergodic and a $B$-process by demonstrating the equivalence to a Markov chain, analogously to Step 1.2. The initial state distribution of each of the processes $\rho_{t}, t \in \mathbb{N}$, is $M_{t}(k)=\delta(1-\delta)^{k}$ and $M_{t}\left(u_{k}\right)=M_{t}\left(d_{k}\right)=\delta(1-\delta)^{k} / 2$ for those $k \in \mathbb{N}$ for which the corresponding states are defined.

Step 2. Having defined $k_{j}, j \in \mathbb{N}$, we can define the process $\rho$. The construction is given in Step 2a, while in Step $2 \mathrm{~b}$ we show that $\rho$ is stationary ergodic and a $B$-process, by showing that it is the limit of the sequence $\rho_{2 j}, j \in \mathbb{N}$.

Step 2a. The process $\rho$ can be constructed as follows (see Fig. 4). The construction is based on the (non-Markovian) process $m_{\rho}$ that has states $0, \ldots, k_{0}$, $k_{2 j+1}+1, \ldots, k_{2(j+1)}, u_{k_{2 j}+1}, \ldots, u_{k_{2 j+1}}$ and $d_{k_{2 j}+1}, \ldots, d_{k_{2 j+1}}$ for $j \in \mathbb{N}$, along with switch states $S_{2 j}$ and reset states $R_{2 j}$. Each switch $S_{2 j}$ diverts the process to the state $u_{k_{2 j}+1}$ if the switch has value up and to $d_{k_{2 j}+1}$ if it has the value down. The reset $R_{2 j}$ sets $S_{2 j}$ to up with probability $1 / 2$ and to down also with probability $1 / 2$. From each state that is neither a reset nor a switch, the process goes to the next state with probability $1-\delta$ and returns to the state 0 with probability $\delta$ (cf. Step $1 k$ ).

The initial distribution $M_{\rho}$ on the states of $m_{\rho}$ is defined as follows. For every state $i$ such that $0 \leq i \leq k_{0}$ and $k_{2 j+1}<i \leq k_{2 j+2}, j=0,1, \ldots$, define the initial probability of the state $i$ as $M_{\rho}(i)=\delta(1-\delta)^{i}$ (the same as in the chain $m_{0}$ ), and for the sets $u_{j}$ and $d_{j}$ (for those $j$ for which these sets are defined), let $M_{\rho}\left(u_{j}\right)=$ 


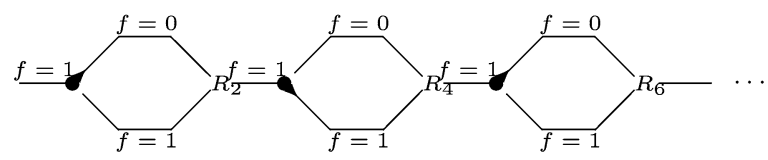

Fig. 4 The processes $m_{\rho}$ and $\rho$. The states are on horizontal lines. The function $f$ being applied to the states of $m_{\rho}$ defines the process $\rho$. Its value is 0 on the states on the upper lines (states $u_{k_{2 j}+1}, \ldots, u_{k_{2 j+1}}$, where $k \in \mathbb{N}$ ) and 1 on the rest of the states

$M_{\rho}\left(d_{j}\right):=\delta(1-\delta)^{i} / 2$ (that is, $1 / 2$ of the probability of the corresponding state of $\left.m_{0}\right)$.

The function $f$ is defined as 1 everywhere except for the states $u_{j}$ (for all $j \in \mathbb{N}$ for which $u_{j}$ is defined) on which $f$ takes the value 0 . The process $\rho$ is defined at time $t$ as $f\left(s_{t}\right)$, where $s_{t}$ is the state of $m_{\rho}$ at time $t$.

Step $2 b$. To show that $\rho$ is a $B$-process, let us first show that it is stationary. Recall Definition 3 of the distributional distance between (arbitrary) process distributions. The set of all stochastic processes, equipped with this distance, is complete, and the set of all stationary processes is its closed subset [3]. Thus, to show that the process $\rho$ is stationary, it suffices to show that $\lim _{j \rightarrow \infty} d\left(\rho_{2 j}, \rho\right)=0$, since the processes $\rho_{2 j}$, $j \in \mathbb{N}$, are stationary. To do this, it is enough to demonstrate that

$$
\lim _{j \rightarrow \infty}\left|\rho\left(\left(x_{1}, \ldots, x_{|B|}\right)=B\right)-\rho_{2 j}\left(\left(x_{1}, \ldots, x_{|B|}\right)=B\right)\right|=0
$$

for each $B \in X^{*}$. Since the processes $m_{\rho}$ and $m_{2 j}$ coincide on all states up to $k_{2 j+1}$, we have

$$
\begin{aligned}
\left|\rho\left(x_{n}=a\right)-\rho_{2 j}\left(x_{n}=a\right)\right| & =\left|\rho\left(x_{1}=a\right)-\rho_{2 j}\left(x_{1}=a\right)\right| \\
& \leq \sum_{k>k_{2 j+1}} M_{\rho}(k)+\sum_{k>k_{2 j+1}} M_{2 j}(k)
\end{aligned}
$$

for all $n \in \mathbb{N}$ and $a \in X$. Moreover, for any tuple $B \in X^{*}$, we obtain

$$
\begin{aligned}
& \left|\rho\left(\left(x_{1}, \ldots, x_{|B|}\right)=B\right)-\rho_{2 j}\left(\left(x_{1}, \ldots, x_{|B|}\right)=B\right)\right| \\
& \leq|B|\left(\sum_{k>k_{2 j+1}} M_{\rho}(k)+\sum_{k>k_{2 j+1}} M_{2 j}(k)\right) \rightarrow 0,
\end{aligned}
$$

where the convergence follows from $k_{2 j} \rightarrow \infty$. We conclude that (6) holds true, so that $d\left(\rho, \rho_{2 j}\right) \rightarrow 0$, and $\rho$ is stationary.

To show that $\rho$ is a $B$-process, we will demonstrate that it is the limit of the sequence $\rho_{2 k}, k \in \mathbb{N}$, in the $\bar{d}$ distance (which was only defined for stationary processes). Since the set of all $B$-process is a closed subset of all stationary processes, it will follow that $\rho$ itself is a $B$-process. (Observe that this way we get the ergodicity of $\rho$ "for free," since the set of all ergodic processes is closed in $\bar{d}$ distance, and all the processes $\rho_{2 j}$ are ergodic.) In order to show that $\bar{d}\left(\rho, \rho_{2 k}\right) \rightarrow 0$, we 
have to find for each $j$ a processes $v_{2 j}$ on pairs $\left(x_{1}, y_{1}\right),\left(x_{2}, y_{2}\right), \ldots$ such that $x_{i}$ are distributed according to $\rho, y_{i}$ are distributed according to $\rho_{2 j}$, and such that $\lim _{j \rightarrow \infty} v_{2 j}\left(x_{1} \neq y_{1}\right)=0$. Construct such a coupling as follows. Consider the chains $m_{\rho}$ and $m_{2 j}$ that start in the same state (with initial distribution being $M_{\rho}$ ) and always take state transitions together, where if the process $m_{\rho}$ is in the state $u_{t}$ or $d_{t}$, $t \geq k_{2 j+1}$ (that is, one of the states that the chain $m_{2 j}$ does not have), then the chain $m_{2 j}$ is in the state $t$. The first coordinate of the process $v_{2 j}$ is obtained by applying the function $f$ to the process $m_{\rho}$, and the second by applying $f_{2 j}$ to the chain $m_{2 j}$. Clearly, the distribution of the first coordinate is $\rho$, and the distribution of the second is $\rho_{2 j}$. Since the chains start in the same state and always take state transitions together, and since the chains $m_{\rho}$ and $m_{2 j}$ coincide up to the state $k_{2 j+1}$, we have $\nu_{2 j}\left(x_{1} \neq y_{1}\right) \leq \sum_{k>k_{2 j+1}} M_{\rho}(k) \rightarrow 0$. Thus, $\bar{d}\left(\rho, \rho_{2 j}\right) \rightarrow 0$, so that $\rho$ is a $B$-process.

Step 3. Finally, it remains to show that the expected output of the test $D$ diverges if the test is run on two independent samples produced by $\rho$.

Recall that for all the chains $m_{2 j}, m_{u 2 j+1}$, and $m_{d 2 j+1}$ as well as for the chain $m_{\rho}$, the initial probability of the state 0 is $\delta$. By construction, if the process $m_{\rho}$ starts at the state 0 , then up to the time step $k_{2 j}$ it behaves exactly as $\rho_{2 j}$ that has started at the state 0 . In symbols, we have

$$
E_{\rho \times \rho}\left(D_{t_{2 j}} \mid s_{0}^{x}=0, s_{0}^{y}=0\right)=E_{\rho_{2 j} \times \rho_{2 j}}\left(D_{t_{2 j}} \mid s_{0}^{x}=0, s_{0}^{y}=0\right)
$$

for $j \in \mathbb{N}$, where $s_{0}^{x}$ and $s_{0}^{y}$ denote the initial states of the processes generating the samples $x$ and $y$, respectively.

We will use the following simple decomposition:

$$
\mathbf{E}\left(D_{t_{j}}\right)=\delta^{2} \mathbf{E}\left(D_{t_{j}} \mid s_{0}^{x}=0, s_{0}^{y}=0\right)+\left(1-\delta^{2}\right) \mathbf{E}\left(D_{t_{j}} \mid s_{0}^{x} \neq 0 \text { or } s_{0}^{y} \neq 0\right) .
$$

From this, (7), and (4) we have

$$
\begin{aligned}
\mathbf{E}_{\rho \times \rho}\left(D_{t_{2 j}}\right) & \leq \delta^{2} \mathbf{E}_{\rho \times \rho}\left(D_{t_{2 j}} \mid s_{0}^{x}=0, s_{0}^{y}=0\right)+\left(1-\delta^{2}\right) \\
& =\delta^{2} \mathbf{E}_{\rho_{2 j} \times \rho_{2 j}}\left(D_{t_{2 j}} \mid s_{0}^{x}=0, s_{0}^{y}=0\right)+\left(1-\delta^{2}\right) \\
& \leq \mathbf{E}_{\rho_{2 j} \times \rho_{2 j}}+\left(1-\delta^{2}\right)<\varepsilon+\left(1-\delta^{2}\right) .
\end{aligned}
$$

For odd indices, if the process $\rho$ starts at the state 0 , then (from the definition of $t_{2 j+1}$ ) by the time $t_{2 j+1}$ it does not reach the reset $R_{2 j}$; therefore, in this case the value of the switch $S_{2 j}$ does not change up to the time $t_{2 j+1}$. Since the definition of $m_{\rho}$ is symmetric with respect to the values up and down of each switch, the probability that two samples $x_{1}, \ldots, x_{t_{2 j+1}}$ and $y_{1}, \ldots, y_{t_{2 j+1}}$ generated independently by (two runs of) the process $\rho$ produce different values of the switch $S_{2 j}$ when passing through it for the first time is $1 / 2$. In other words, with probability $1 / 2$ two samples generated by $\rho$ starting at the state 0 will look by the time $t_{2 j+1}$ as two samples generated by $\rho_{u 2 j+1}$ and $\rho_{d 2 j+1}$ that have started at state 0 . Thus,

$$
E_{\rho \times \rho}\left(D_{t_{2 j+1}} \mid s_{0}^{x}=0, s_{0}^{y}=0\right) \geq \frac{1}{2} E_{\rho_{u 2 j+1} \times \rho_{d 2 j+1}}\left(D_{t_{2 j+1}} \mid s_{0}^{x}=0, s_{0}^{y}=0\right)
$$


for $j \in \mathbb{N}$. Using this, (8), and (5), we obtain

$$
\begin{aligned}
\mathbf{E}_{\rho \times \rho}\left(D_{t_{2 j+1}}\right) & \geq \delta^{2} \mathbf{E}_{\rho \times \rho}\left(D_{t_{2 j+1}} \mid s_{0}^{x}=0, s_{0}^{y}=0\right) \\
& \geq \frac{1}{2} \delta^{2} \mathbf{E}_{\rho_{2 j+1} \times \rho_{2 j+1}}\left(D_{t_{2 j+1}} \mid s_{0}^{x}=0, s_{0}^{y}=0\right) \\
& \geq \frac{1}{2}\left(\mathbf{E}_{\rho_{2 j+1} \times \rho_{2 j+1}}\left(D_{t_{2 j+1}}\right)-\left(1-\delta^{2}\right)\right)>\frac{1}{2}\left(\delta^{2}-\varepsilon\right) .
\end{aligned}
$$

Taking $\delta$ large and $\varepsilon$ small (e.g., $\delta=0.9$ and $\varepsilon=0.1$ ), we can make the bound (9) close to 0 and the bound (11) close to $1 / 2$, and the expected output of the test will cross these values infinitely often. Therefore, we have shown that the expected output of the test $D$ diverges on two independent runs of the process $\rho$, contradicting the consistency of $D$. This contradiction concludes the proof.

Acknowledgements The author is grateful to the anonymous reviewer for several remarks that have led to the improvement of the paper. This research has been partially supported by French National Research Agency (ANR), project ANR-08-COSI-004.

\section{References}

1. Adams, T.M., Nobel, A.B.: On density estimation from ergodic processes. Ann. Probab. 26(2), 794804 (1998)

2. Gyorf, L., Morvai, G., Yakowitz, S.: Limits to consistent on-line forecasting for ergodic time series. IEEE Trans. Inf. Theory 44(2), 886-892 (1998)

3. Gray, R.: Probability, Random Processes, and Ergodic Properties. Springer, Berlin (1988)

4. Lehmann, E.L.: Testing Statistical Hypotheses. Springer, Berlin (1986)

5. Morvai, G., Weiss, B.: On classifying processes. Bernoulli 11(3), 523-532 (2005)

6. Nobel, A.B.: Hypothesis testing for families of ergodic processes. Bernoulli 12(2), 251-269 (2006)

7. Ornstein, D.S.: Ergodic Theory, Randomness, and Dynamical Systems. Yale Mathematical Monographs, vol. 5. Yale Univ. Press, New Haven (1974)

8. Ornstein, D.S., Shields, P.: The $\bar{d}$-recognition of processes. Adv. Math. 104, 182-224 (1994)

9. Ornstein, D.S., Weiss, B.: How sampling reveals a process. Ann. Probab. 18(3), 905-930 (1990)

10. Ryabko, B.: Prediction of random sequences and universal coding. Probl. Inf. Transm. 24, 87-96 (1988)

11. Ryabko, B., Astola, J., Gammerman, A.: Application of Kolmogorov complexity and universal codes to identity testing and nonparametric testing of serial independence for time series. Theor. Comput. Sci. 359, 440-448 (2006)

12. Ryabko, D., Ryabko, B.: Nonparametric statistical inference for ergodic processes. IEEE Trans. Inf. Theory (to appear)

13. Ryabko, D., Ryabko, B.: On hypotheses testing for ergodic processes. In: Proceedings of IEEE Information Theory Workshop (ITW'08), Porto, Portugal, pp. 281-283 (2008)

14. Shields, P.: Two divergence-rate counterexamples. J. Theor. Probab. 6, 521-545 (1993)

15. Shields, P.: The interactions between ergodic theory and information theory. IEEE Trans. Inf. Theory 44(6), 2079-2093 (1998)

16. Shields, P.: The Ergodic Theory of Discrete Sample Paths. AMS Bookstore. AMS, Providence (1996)

17. Shiryaev, A.: Probability, 2nd edn. Springer, Berlin (1996) 